For reprint orders, please contact: reprints@futuremedicine.com

\title{
Emerging immune therapy of metastatic melanoma in the older patient: does age really matter?
}
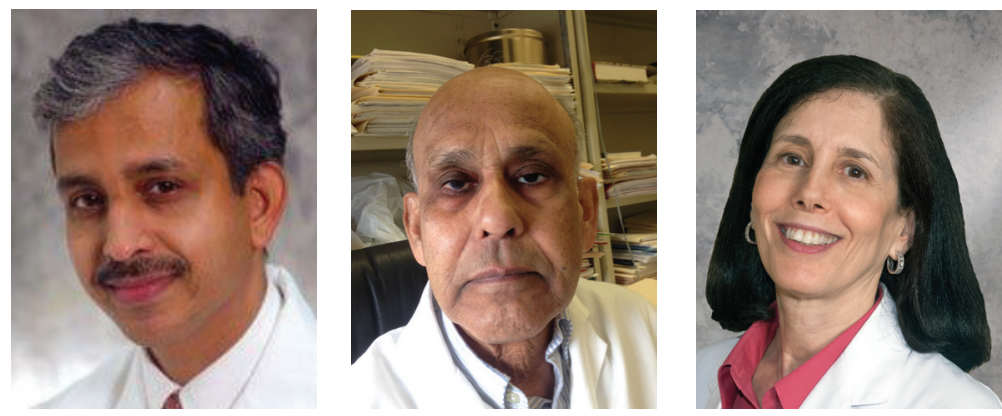

Upendra P Hegde ${ }^{*, 1,2}$, Bijay Mukherji' \& Jane M Grant-Kels ${ }^{1}$

First draft submitted: 12 October 2015; Accepted for publication: 12 October 2015; Published online: 17 February 2016

Melanoma incidence is rapidly increasing in the geriatric population making it a major public health issue [1,2]. Healthcare cost analysis of cancer treatment suggests a significant economic burden related to metastatic melanoma treatment of the elderly patient [3]. Larger studies have shown that age is an independent negative prognostic factor of survival in older subjects diagnosed with cutaneous melanoma [4]. Melanoma is an immunogenic tumor and anti-melanoma immunity plays an important role in shaping its course as well as the rising incidence noted in the geriatric population [5]. Given the aging population is rapidly expanding and recent T-cell checkpoint blockade-based immunotherapies have shown remarkable effectiveness, it is becoming increasingly important to understand whether or not elderly melanoma patients should be treated with these agents.

It has been acknowledged for a long time that systemic chemotherapy does not improve survival in patients diagnosed with metastatic melanoma [6]. In general, elderly cancer patients are not often included in clinical trials as well as not always treated with potentially effective systemic treatments. This is generally because elderly patients have been found to be less responsive to anticancer therapy and they do not usually tolerate the toxicities associated with systemic therapies. Historically, melanoma treatment in the elderly lacked enthusiastic appeal from physicians, patients and patients' families due to inefficacy and toxicity associated with the available treatments.

Reports of spontaneous regression observed in patients diagnosed with metastatic melanoma suggest that antimelanoma immunity likely plays a role [7]. The discovery of IL-2 as a first T-cell tropic factor and its consistent clinical efficacy in a subgroup of patients diagnosed with metastatic melanoma led to its approval by the US FDA in 1998 [8]. Unfortunately, life-threatening toxicity due to the capillary leak associated with high-dose IL-2 
"Accrual of increasing numbers of older patients in immune therapy-based clinical trials will help develop valuable insights into the role of immuneenhancing therapy in the setting of age-associated deficiencies of the immune system." discouraged its use in older patients who were routinely excluded from clinical trials containing this agent due to their co-morbidities and concerns of toxicity [9]. Reports of older subjects diagnosed with renal cell carcinoma responding to treatment with high-dose IL-2 indicated that $\mathrm{T}$ cells, although weakened in older subjects, are able to be activated by IL-2 [10]. The laboratory observation of lymphocytes clustered around melanoma tumor cells led to the identity of melanoma epitopes and epitope-specific effector $T$ cells [11,12]. This intensified the interest in melanoma vaccines. Encouraged by animal data, a number of human melanoma peptide vaccine trials were designed and completed. Unfortunately, the responses were inconsistent and unpredictable [13]. The realization that among other causes of vaccine failure tumor sensitized effector $T$ cells are seriously constrained by their expression of inhibitory molecules such as CTLA- 4 and PD- 1 has re-ignited the interest in immune therapy of cancer through design of monoclonal antibodies capable of blocking the inhibitory molecules [14].

Melanoma as a model of an immunogenic tumor provides a unique opportunity to understand the clinical relevance of immune checkpoint inhibitor treatment in the elderly population. Emerging immunotherapeutic strategies that activate effector $\mathrm{T}$ cells through blockade of CTLA- 4 and PD-1 receptors and their ligands are showing promising results in patients. In 2011, based on the results of large Phase III clinical studies, the US FDA-approved ipilimumab as the first monoclonal antibody against T-cell inhibitory molecule CTLA-4. In 2014 an antiPD-1 agent Pembrolizumab was approved for use in patients diagnosed with metastatic melanoma who previously failed anti-CTLA- 4 agent $[15,16]$. Both studies included patients below and over 65 years of age with survival benefit seen in each of these groups.

The issue of elderly melanoma patients and emerging T-cell activating treatment strategies has other considerations as the immune checkpoint blockade-based therapies for melanoma involves harnessing one's own anti-melanoma immunity and aging is associated with 'senescence'. Indeed the literature is replete with reports of elderly cancer patients not doing well with anticancer therapies due to T-cell weaknesses and imbalances within their immune regulatory apparatus. Thus clinicians now have a dilemma in making therapeutic decision for elderly melanoma patients in the context of checkpoint blockade-based therapies. Given that these therapies are now approved therapies and as more and more elderly patients with melanoma are likely to seek the 'best available treatment', we are of the opinion that before elderly melanoma patients are denied current forms of immunotherapies, the question of to treat or not to treat elderly melanoma patients with checkpoint blockade-based reagents needs to be made after significant deliberation. In our judgment, the following considerations must be addressed:

- With advances in healthcare delivery and improved lifestyles, people are living longer. Even with co-morbidities, the quality of life of the elderly has improved resulting in improved life expectancy. As such, it is important to make available to the older subjects the promising treatments available to their younger counterparts;

- Just as not all young patients with melanoma do not do well with cancer and do not tolerate systemic anticancer therapies well, all elderly patients with melanoma do not do poorly and some 'well preserved' elderly patients might tolerate side effects reasonably well;

- Aging can no longer be lumped into a single entity based on a metric of numerical age. A significant proportion of older subjects with our without co-morbidities are physically fit enough to be considered for treatment of metastatic melanoma. A recent analysis of the data obtained from the Connecticut Cancer Registry examined the treatment outcome of older metastatic melanoma patients using a competing morbidity model; the results showed no difference in outcomes between young and older subjects [17];

- As pointed out earlier, checkpoint blockadebased melanoma immunotherapies aims at re-engaging the $\mathrm{T}$-cell responses by taking the 'brake off.' In this context, although the elderly may not be capable of making normal quantities of new $T$ cells due to thymic involution, they might harbor a fair number (possibly even larger numbers of antigen-experienced $T$ cells) of melanoma reactive $T$ cells. This, therefore, might make them potentially more responsive to this form of therapy. However, more data and experience is required to answer whether the clinical responses to 
immune checkpoint inhibitors in older subjects diagnosed with metastatic melanoma will be durable due to the aging-associated exhaustion of the T cells;

- Immune senescence in the elderly is well established. Yet when T cells from the elderly and the young cohort are tested for responsiveness with the $\mathrm{T}$ cells from both cohorts made to bear an identical set of receptors and made to react to a single epitope, the $\mathrm{T}$ cells from the elderly do not appear functionally inferior [18];

- The effector versus regulatory T-cell network might be out of balance in the elderly. However, just as effector $\mathrm{T}$ cells express co-inhibitory receptors, regulatory $\mathrm{T}$ cells also express them [19]. As such, the effect of checkpoint blockade-based therapies in the elderly might have a different connotation. This issue, however, needs careful examination;

- The issue of treatment tolerance by the elderly is a legitimate concern. Elderly subjects are prone to develop autoimmunity. Therefore it is likely that autoimmune manifestations might be amplified following immune checkpoint inhibitor therapy of metastatic melanoma. Since suppression of the immune function remains the cornerstone of managing immune-related adverse events and the elderly do not tolerate immune suppression well, additional challenges will be faced to accomplish safe delivery of these agents in the elderly compared with their younger counterparts. Furthermore, the physical as well as social limitations associated with older age often reduce effective communication between healthcare providers and the elderly patient that is critical to safe delivery of these agents [20]. However, in the absence of solid data on dose/response/ toxicities, treatment decision has to be made based upon 'good clinical practice';
- Finally, we recognize that the cost of these new therapies required to extend the life of a geriatric patient with metastatic melanoma has implications regarding the rising cost of medical care and the limited resources available. Nonetheless, it is our opinion that until critical data on cost-benefit parameters of treatment of elderly melanoma patients with these forms of costly medicine is available, the decision of 'to treat or not to treat' an elderly melanoma patient with expensive but potentially life prolonging treatment with immune checkpoint blockade-based therapies has to be made jointly by the doctor as well as the patient and their family.

In summary, early results show that older patients do share the benefits of immune checkpoint inhibitors. More work will be needed to understand if these benefits are uniformly shared by all across the aging spectrum that include the oldest of the old. It is difficult and ethically challenging to deprive older metastatic melanoma patients the optimal treatment that is available to their younger counterparts. Safety and predictable benefits are equally important considerations that deserve further studies. Accrual of increasing numbers of older patients in immune therapybased clinical trials will help develop valuable insights into the role of immune-enhancing therapy in the setting of age-associated deficiencies of the immune system.

Financial \& competing interests disclosure

This work was supported by a philanthropic gift to UP Hedge from Jane and Richard Lublin. The authors have no other relevant affiliations or financial involvement with any organization or entity with a financial interest in or financial conflict with the subject matter or materials discussed in the manuscript apart from those disclosed.

No writing assistance was utilized in the production of this manuscript.

\section{"' It is difficult and ethically challenging to deprive \\ older metastatic melanoma patients the optimal \\ treatment that is available to their younger counterparts."}

\section{References}

1 Cancer Facts and Figures 2010. American Cancer Society, Atlanta, GA, USA (2010).

2 Geller AC, Miller DR, Annas GD et al. Melanoma incidence and mortality among US whites, 1969-1999. JAMA 288, 1719-1720 (2002).

3 Seidler AM, Pennie ML, Veledar E et al. Economic burden of melanoma in the elderly population. Arch. Dermatol. 146(3), 249-256 (2010).
4 Balch CM, Gershenwald JE, Soong SJ et al. Final version of 2009 AJCC melanoma staging and classification. J. Clin. Oncol. 36, 6199-6206 (2009).

5 Hegde UP, Chakraborty NG, Kerr P, Grant-Kels JM. Melanoma in the elderly patient: relevance of the aging immune system. Clin. Dermatol. 27, 537-544 (2009).

6 Eigentler TK, Caroli UM, Radny P, Garbe C. Palliative therapy of disseminated melanoma: a systematic review of 41 randomized clinical trials. Lancet Oncol. 4(12), 748-759 (2003).
7 Smith JL, Stehlin JS. Spontaneous regression of primary melanoma with regional metastases. Cancer 18, 1399-1415 (1965).

8 Atkins MB, Lotz MT, Dutcher JP et al. Highdose recombinant interleukin2 therapy for patients with metastatic melanoma: analysis of 270 patients treated between 1985 and 1993. J. Clin. Oncol. 17, 2105-2116 (1999).

9 Kammula US, White DE, Rosenberg SA. Trends in the safety of high dose bolus interleukin-2 administration in patients with metastatic cancer. Cancer 15, 22-28 (1998). 
10 Quan W Jr. Ramirez M, Taylor C et al. Administration of high-dose IL-2 to patients age 70 or over. Cancer Biother. Radiopharm. 20, 11-15 (2005)

11 Mukherji B, Chakraborty NG, Yamasaki S et al. Induction of antigen-specific cytolytic $\mathrm{T}$ cells in situ in human melanoma by immunization with synthetic peptide pulsed autologous antigen presenting cells. Proc. Natl Acad. Sci. USA 92, 8078-8082 (1995).

12 Boon T, Coulie PG, Van den Eynde BJ et al. Human $T$ cell responses against melanoma. Ann. Rev. Immunol. 24, 1-34 (2006).

13 Rosenberg SA, Yang JC, Restifo NP. Cancer immunotherapy: moving beyond current vaccines. Nat. Med. 10(9), 909-915 (2004).

14 Van Elsas A, Hurwitz AA, Allison JP. Combination immunotherapy of B16 melanoma using anti-cytotoxic T lymphocyte-associated antigen 4
(CTLA-4) and granulocyte/macrophage colony-stimulating factor (GM-CSF)producing vaccines induces rejection of subcutaneous and metastatic tumors accompanied by autoimmune pigmentation. J. Exp. Med. 190 (3), 355-366 (1999).

15 Hodi FS, O’Day SJ, McDermott DF et al. Improved survival with ipilimumab in patients with metastatic melanoma. N. Engl. J. Med. 363, 711-723 (2010).

16 Ribas A, Hodi SF, Kefford R et al. Efficacy and safety of the anti-PD-1 monoclonal antibody MD-3475 in 411 patients with melanoma. Presented at: 2014 American Society of Clinical Oncology Annual Meeting. Chicago, IL, USA, 30 May-3 June 2014.

17 Hoag J, Hegde UP, Zweifler R, Berwick $\mathrm{M}$, Swede $\mathrm{H}$. Age as a prognostic factor of stage IV melanoma: a competing risks analysis using proportional sub distribution hazards regression. Presented at: American Public Health Association 143rd Annual Meeting. Chicago, IL, USA,

31 October-4 November 2015.

18 Chakraborty NG, Yadav M, Dadras SS et al. Analyses of T cell-mediated immune response to a human melanoma-associated antigen by the young and the elderly. Hum. Immunol. 74(5), 640-647 (2013).

19 Hegde UP, Chakraborty N, Mukherji B, Grant-Kels JM. Metastatic melanoma in the older patient: immunologic insights and treatment outcomes. Expert Rev. Pharmacoecon. Outcomes Res. 11, 185-193 (2011).

20 Weber JS, Kahler KC, Hauschild A. Management of immune-related adverse events and kinetics of response with ipilimumab. J. Clin. Oncol. 21, 2691-2697 (2012). 